and learned behaviours are the breakdown of community norms ${ }^{3}$ such as lack of family cohesion, lack of a social support network, dysfunctional families and child abuse. Also, in high-income countries as people enjoy more privileges, they tend to take less responsibility for their actions and expect more and more from the state. We increasingly see more pressure on social services, rather than on parents, to account for the welfare of children.

This does not mean that borderline personality disorder is exclusive to the West, but in the social context we do see more reasons for people in the West to have such traits.

Given the aetiological factors that we are aware of, and the crucial age factor for borderline personality disorder, it is no surprise that immigration is not a risk factor for borderline personality disorder.

This is an interesting study that confirms what was earlier suggested by Tyrer et $a l^{4}$ and Baleydier et al; ${ }^{5}$ however, I am not sure whether a similar study in future would be useful, given that it is unlikely that immigration can be a risk factor for developing borderline personality disorder.

I do, however, agree with the authors that future studies in younger immigrants and second generations who will be more influenced by the Western way of life are likely to be interesting and helpful, especially in terms of clinical management.

1 Pascual JC, Malagón A, Córcoles D, Ginés JM, Soler J, García-Ribera C, et al. Immigrants and borderline personality disorder at a psychiatric emergency service. Br J Psychiatry 2008; 193: 471-6.

2 Millon T. Sociocultural conceptions of the borderline personality. Psychiatr Clin North Am 2000; 23: 123-36.

3 Paris J. Social Factors in the Personality Disorders: A Biopsychosocial Approach to Etiology and Treatment. Cambridge University Press, 1996.

4 Tyrer $\mathrm{P}$, Merson S, Onyett S, Johnson $\mathrm{T}$. The effects of personality disorder on clinical outcome, social networks and adjustment: a controlled clinical trial of psychiatric emergencies. Psychol Med 1994; 24: 731-40.

5 Baleydier B, Damsa C, Schutzbach C, Stauffer O, Glauser D. Comparison between Swiss and foreign patients' characteristics at the psychiatric emergencies department and the predictive factors of their management strategies. Encephale 2003; 29: 205-12.

Salman A. Mushtaq, Crisis Resolution Home Treatment, Swanswell Point, Stoney Stanton Road, Coventry CV1 4FH, UK. Email: ghazalizee@hotmail.com

doi: 10.1192/bjp.194.5.467b

Authors' reply: We thank Dr Mushtaq for his comments on our article. ${ }^{1}$ Although we agree with his comment that it is unlikely that immigration could be a risk factor for developing borderline personality disorder, we think that this issue is still open to debate.

First, other authors such as Paris $^{2}$ have suggested that the process of migration from traditional societies to Western countries could result in the development of borderline personality disorder in individuals who did not present any symptoms in their country of origin. Paris considered that although individuals could have a biological predisposition to this disorder, such as an innate affective instability, the structure of traditional societies tends to suppress the kind of psychopathology seen in borderline personality disorder. Once these patients emigrate to Western countries, this sociocultural suppression disappears. ${ }^{2}$ In contrast, Tyrer et $a l^{3}$ and Baleydier et $a l^{4}$ observed a lower incidence of personality disorders in immigrant patients admitted to psychiatric emergency services. Likewise, in a previous study that was not centred on an immigrant population, we found that patients with borderline personality disorder were less likely to be immigrants. ${ }^{5}$ For this reason, we performed an exploratory study (i.e. without an initial hypothesis) to examine whether there really was an association between immigration and borderline personality disorder, where immigration could either be a risk factor or have a 'protective' effect. ${ }^{1}$ Despite the fact that, in our opinion, we observed a 'protective' association for immigration on the development of borderline personality disorder, our results do not invalidate Paris's hypothesis. In Spain, immigration is a relatively new phenomenon, and the majority of patients we evaluated were adults from poorer countries who were not yet totally immersed in Western culture. It is possible that in younger immigrants (whose personality has not yet been totally consolidated) or in second-generation immigrants, a higher prevalence of borderline personality disorder could eventually be observed, as suggested by Paris. ${ }^{2}$

Second, another important point of our study is that the immigrant sample must not be considered as a homogeneous group, since important differences exist between the subgroups of immigrants according to their geographical origin. For instance, patients from sub-Saharan Africa and Asian countries were more than seven times less likely than other immigrants to be diagnosed with borderline personality disorder. Therefore, it could be suggested that certain cultural differences in these regions, for example a greater tolerance of suffering, could be useful factors to prevent the development of this disorder. The identification and analysis of these 'protective' cultural factors could offer future tools to prevent the appearance of borderline personality disorder in Western societies.

We would also like to highlight that although we share Dr Mushtaq's opinion that it is unlikely that immigration may be a risk factor for borderline personality disorder, the empirical evidence so far is not only scarce but also somewhat contradictory and with important methodological limitations. In fact, our own study presents some of these limitations. To confirm our findings, more methodologically rigorous studies would be necessary.

1 Pascual JC, Malagón A, Córcoles D, Ginés JM, Soler J, García-Ribera C, et al. Immigrants and borderline personality disorder at a psychiatric emergency service. Br J Psychiatry 2008; 193: 471-6.

2 Paris J. Cultural factors in the emergence of borderline pathology. Psychiatry 1996; 59: 185-92.

3 Tyrer $\mathrm{P}$, Merson $\mathrm{S}$, Onyett $\mathrm{S}$, Johnson $\mathrm{T}$. The effects of personality disorder on clinical outcome, social networks and adjustment: a controlled clinical trial of psychiatric emergencies. Psychol Med 1994; 24: 731-40.

4 Baleydier B, Damsa C, Schutzbach C, Stauffer O, Glauser D. Comparison between Swiss and foreign patients' characteristics at the psychiatric emergencies department and the predictive factors of their management strategies. Encephale 2003; 29: 205-12.

5 Pascual JC, Córcoles D, Castaño J, Ginés JM, Gurrea A, Martín-Santos R, et al. Hospitalization and pharmacotherapy for borderline personality disorder in a psychiatric emergency service. Psychiatr Serv 2007; 58: 1199-204.

Juan C. Pascual, Department of Psychiatry, Hospital de la Santa Creu i Sant Pau, Universitat Autònoma de Barcelona, and CIBER-SAM, Barcelona, Spain. Email: jpascual@santpau.es; Joaquim Soler, víctor Pérez, Department of Psychiatry, Hospital de la Santa Creu i Sant Pau, Universitat Autònoma de Barcelona, and CIBER-SAM, Barcelona, Spain

doi: 10.1192/bjp.194.5.468

\section{Clozapine and risk of pneumonia}

Taylor et al showed that among the 'reasons for discontinuing' clozapine is the unfortunate outcome of death. ${ }^{1}$ Out of the 21 deaths reported, five patients died from pneumonia ( 24\%). Interestingly, 'there was no evidence of neutropenia or agranulocytosis in any patients at the time of death. ${ }^{1}$

The relationship between clozapine and infection is indeed complex. Links between clozapine agranulocytosis, and between agranulocytosis and the increased risk of infection are well 